PROCEEDINGS OF THE

AMERICAN MATHEMATICAL SOCIETY

Volume 133, Number 2, Pages 449-453

S 0002-9939(04)07627-0

Article electronically published on August 30, 2004

\title{
A VERSION OF SHARKOVSKII'S THEOREM FOR DIFFERENTIAL EQUATIONS
}

\author{
JAN ANDRES AND KAREL PASTOR \\ (Communicated by Carmen C. Chicone)
}

\begin{abstract}
We present a version of the Sharkovskii cycle coexistence theorem for differential equations. Our earlier applicable version is extended here to hold with the exception of at most two orbits. This result, which (because of counter-examples) cannot be improved, is then applied to ordinary differential equations and inclusions. In particular, if a time-periodic differential equation has $n$-periodic solutions with $n \neq 2^{m}$, for all $m \in \mathbb{N}$, then infinitely many subharmonics coexist.
\end{abstract}

\section{Standard Sharkovskit's theorem does not APPly TO DIFFERENTIAL EQUATIONS}

The celebrated Sharkovskii cycle coexistence theorem [Sh], based on a new ordering of the positive integers, namely

$$
\begin{gathered}
3 \triangleright 5 \triangleright 7 \triangleright \cdots \triangleright 2 \cdot 3 \triangleright 2 \cdot 5 \triangleright 2 \cdot 7 \triangleright \cdots \triangleright 2^{2} \cdot 3 \triangleright 2^{2} \cdot 5 \triangleright 2^{2} \cdot 7 \triangleright \\
\cdots \triangleright 2^{n} \cdot 3 \triangleright 2^{n} \cdot 5 \triangleright 2^{n} \cdot 7 \triangleright \cdots \triangleright 2^{n+1} \cdot 3 \triangleright 2^{n+1} \cdot 5 \triangleright 2^{n+1} \cdot 7 \triangleright \\
\cdots \triangleright 2^{n+1} \triangleright 2^{n} \triangleright \cdots \triangleright 2^{2} \triangleright 2 \triangleright 1,
\end{gathered}
$$

reads as follows:

Theorem 1 (A. N. Sharkovskii). Let $f: \mathbb{R} \rightarrow \mathbb{R}$ be a continuous function. If $f$ has a point of period $n$ with $n \triangleright k$ (in the above Sharkovskii ordering), then it has also a point of period $k$.

By a period, we mean the least period, i.e. a point $a \in \mathbb{R}$ is a periodic point of period $n$ of $f$ if $f^{n}(a)=a$ and $f^{j}(a) \neq a$, for $0<j<n$.

Consider the scalar (e.g. continuous) differential equation

$$
x^{\prime}=F(t, x), \text { where } F(t, x) \equiv F(t+1, x),
$$

and assume that all its solutions extend to $(-\infty, \infty)$ and that the corresponding initial value problems are uniquely solvable. It is well known that uniqueness implies continuous dependence on initial values; hence, the related Poincaré translation operators $(m \in \mathbb{N})$

$$
\Phi_{m}\left(x_{0}\right):=\left\{x\left(m, x_{0}\right) \mid x\left(\cdot, x_{0}\right) \text { is a solution of (11) with } x\left(0, x_{0}\right)=x_{0}\right\}
$$

Received by the editors September 3, 2003.

2000 Mathematics Subject Classification. Primary 34C25, 34A60, 37E05, $47 \mathrm{H} 04$.

Key words and phrases. Sharkovskii's theorem, applicable (multivalued) version, $M$-maps, (primary) orbits, translation operators, subharmonics, multiplicity results.

Supported by the Council of Czech Government (J14/98:153100011). 
are completely continuous. In spite of the apparent one-to-one correspondence between periodic points of $\Phi_{1}$ and subharmonic solutions of (1), the analogue of Theorem 11 does not hold for subharmonics of (1). In fact, we only obtain an empty statement because every bounded solution of (1) is, under the uniqueness assumption, either 1-periodic or asymptotically 1-periodic (see e.g. [Pl, pp. 120-122]).

\section{A VERsion of Sharkovskit's THEOREM THAT HOLDS WITH THE EXCEPTION OF AT MOST TWO ORBITS}

Even if the solutions of the initial value problem for system (1) are not always unique, the corresponding Poincaré translation operators $\Phi_{m}=\Phi_{1}^{m}, m \in \mathbb{N}$, are upper-semicontinuous (i.e., for every open $V \subset \mathbb{R}$, the set $\left\{x_{0} \in \mathbb{R} \mid \Phi_{m}\left(x_{0}\right) \subset V\right\}$ is open), and the sets of values of $\Phi_{m}$ consist either of single points or of compact intervals (see $\mathrm{AFJ}$ ). For the sake of simplicity, such multivalued maps (i.e. $\varphi$ : $\mathbb{R} \leadsto \mathbb{R}$ resp. $\varphi: \mathbb{R} \mapsto 2^{\mathbb{R}} \backslash\{\emptyset\}$ that are upper-semicontinuous and whose values are either single points or compact intervals) will be called $M$-maps.

A natural question arises: Can the (single-valued) continuous function $f$ be replaced in Theorem 1 by an $M$-map?

The answer is no, in general. For example, periodic points of period 3 need not imply the existence of periodic points of periods 2 and 4 (see [AFJ]). Here, $a$ is a period $n$ point of the $M$-map $\varphi: \mathbb{R} \leadsto \mathbb{R}$ if $a \in \varphi^{n}(a)$, but $a \notin \varphi^{j}(a)$, for $0<j<n$. But in the multivalued setting, it is more appropriate to consider periodic orbits rather than periodic points because the latter excludes many possibilities.

An orbit of nth-order (or, an $n$-orbit) of an $M$-map $\varphi: \mathbb{R} \leadsto \mathbb{R}$ is a sequence $\left\{x_{i}\right\}_{i=0}^{\infty}$ such that

(i) $\quad x_{i+1} \in \varphi\left(x_{i}\right)$ for $i \geq 0$,

(ii) $\quad x_{i}=x_{i+n}$ for $i \geq 0$, and

(iii) this orbit is not a product orbit formed by going $p$ times around a shorter orbit of $m t h$-order, where $m p=n$.

If, in addition,

(iv) $\quad x_{i} \neq x_{j}$, for $i \neq j ; i, j=0,1, \ldots, n-1$, we say that $\left\{x_{i}\right\}_{i=0}^{\infty}$ is a primary orbit of $n t h$-order (or, a primary $n$-orbit).

Unfortunately, Theorem 1 does not hold for orbits of $M$-maps. For example, the existence of a 3-orbit need not imply the existence of orbits of orders 2 or 4 or 6 (see $[\mathrm{AFJ}, \mathrm{AJP}$ ). On the other hand, Theorem 1 holds for the generic $M$-map with few exceptions.

Proposition 1 ( $\mathrm{AG})$. If an $M$-map has an $n$-orbit, then it has an order- $k$ orbit for every $k \triangleleft n$ with at most three exceptions.

The last result, which had a technically difficult and long proof (see $\mathrm{AG}$ and cf. [AFJ], AJ], AJP]), is lowered to the status of a proposition here because we will improve it to hold with the exception of at most two orbits.

The exceptions mentioned in Proposition 1 can be determined exactly:

Proposition 2 ([AJP $)$. Let an $M-\operatorname{map} \varphi: \mathbb{R} \leadsto \mathbb{R}$ have an $n$-orbit, where $n=$ $2^{m} q, m \in \mathbb{N}_{0}=\mathbb{N} \cup\{0\}, q$ is odd, and $n$ is maximal in the Sharkovskii ordering.

1. If $q>3$, then $\varphi$ has a $k$-orbit for every $k \triangleleft n$, except $k=2^{m+2}$.

2. If $q=3$, then $\varphi$ has a $k$-orbit, for every $k \triangleleft n$, except $k=2^{m+1} \cdot 3$, $2^{m+2}, 2^{m+1}$.

3. If $q=1$, then $\varphi$ has a $k$-orbit, for every $k \triangleleft n$. 
A glance at Proposition 2 and the counter-examples given in AFJ] and AJP] suggests a question: Is there an $M$-map with a 3 -orbit but no orbits of orders 2,4 and 6 . For the answer, we use the following lemma.

Lemma 1 ([AFJ]). Let $\varphi: \mathbb{R} \leadsto \mathbb{R}$ be an $M$-map. Assume that $I_{k} \subset \mathbb{R}, k=$ $0,1, \ldots, n-1$, are closed intervals such that $I_{k+1} \subset \varphi\left(I_{k}\right)$, for $k=0,1, \ldots, n-1$, which we write as $I_{0} \rightarrow I_{1} \rightarrow \cdots \rightarrow I_{n}=I_{0}$. Then the $n$-th iterate $\varphi^{n}$ of $\varphi$ (i.e. the $n$-fold composition of $\varphi$ with itself) has a fixed-point $x_{0}$ (i.e. $x_{0} \in \varphi^{n}\left(x_{0}\right)$ ) with $x_{k+1} \in \varphi\left(x_{k}\right), x_{n}=x_{0}$, where $x_{k} \in I_{k}$, for $k=0,1, \ldots, n-1$.

Proposition 3. Let $\varphi: \mathbb{R} \leadsto \mathbb{R}$ be an $M$-map. If $\varphi$ has a 3-orbit, then at least one of the following two cases occurs:

(i) $\varphi$ has a 2-orbit.

(ii) $\varphi$ has a 4-orbit and a 6-orbit.

Proof. We can assume that the 3 -orbit $\{a, b, c\}$ is primary, i.e. it is formed by three different points. Indeed, if for example $b=c$, then $\varphi$ has the 4 -orbit $\{a, b, b, b\}$ and the 6 -orbit $\{a, b, b, b, b, b\}$. Furthermore, the 3 -orbit $\{a, b, c\}$ can be assumed to satisfy $a<b, a<c$ (otherwise, we rename the points). There are two possibilities.

Case 1. $b<c$.

$$
\text { For }
$$

$$
[a, b] \rightarrow[b, c] \rightarrow[a, b]
$$

we obtain by Lemma 1 that $\varphi$ has either a 2-orbit or $b \in \varphi(b)$. In the latter case, $\varphi$ has the 4 -orbit $\{a, b, b, c\}$ and the 6 -orbit $\{a, b, b, b, b, c\}$.

Case 2. $c<b$.

For

$$
[a, c] \rightarrow[c, b] \rightarrow[a, c]
$$

again apply Lemma 1 to see that $\varphi$ has either a 2-orbit or $c \in \varphi(c)$. In case $c \in \varphi(c), \varphi$ has the 4 -orbit $\{a, b, c, c\}$ and the 6 -orbit $\{a, b, c, c, c, c\}$.

Using Proposition 3 and the following lemmas we will determine the exceptions for the initial numbers of subsequences of the Sharkovskii ordering.

Lemma 2 ( $[\mathrm{AJP}]$ ). Let $\varphi: \mathbb{R} \leadsto \mathbb{R}$ and $g: \mathbb{R} \leadsto \mathbb{R}$ be $M$-maps satisfying $g=\varphi^{l}$, where $l \in \mathbb{N}$, and let $q$ be odd. If $\varphi$ has an lq-orbit, then $g$ has a q-orbit.

Lemma $3(\overline{\mathrm{AJP}})$. Let $\varphi: \mathbb{R} \leadsto \mathbb{R}$ and $g: \mathbb{R} \leadsto \mathbb{R}$ be $M$-maps satisfying $g=\varphi^{l}$, where $l=2^{s}$, for some $s \in \mathbb{N}$.

(1) If $g$ has a q-orbit, where $q$ is odd, then $\varphi$ has also a q-orbit or an lq-orbit.

(2) If $g$ has a $q$-orbit, where $q=2^{r}$, for some $r \in \mathbb{N}$, then $\varphi$ has a $2^{r+s}$-orbit.

Proposition 4. Let $\varphi: \mathbb{R} \leadsto \mathbb{R}$ be an $M$-map, $m \in \mathbb{N}_{0}$. If $\varphi$ has a $3 \cdot 2^{m}$-orbit, then at least one of the following two cases occurs:

(i) $\varphi$ has a $2^{m+1}$-orbit.

(ii) $\varphi$ has a $2^{m+2}$-orbit and a $2^{m+1} \cdot 3$-orbit.

Proof. It follows from Proposition 2 and Proposition 3 that we can assume $\varphi$ has no odd orbit. Let us consider an $M$-map $g: \mathbb{R} \leadsto \mathbb{R}$ such that $g=\varphi^{2^{m}}$. Using Lemma 2, $g$ has a 3-orbit. 
By Lemma 3 (2), the existence of a 2-orbit of $g$ implies the existence of a $2^{m+1}$-orbit of $\varphi$ and the existence of a 4-orbit of $g$ implies the existence of a $2^{m+2}$ orbit of $\varphi$. Thus, in view of Proposition [3, it suffices to show that the existence of a 6-orbit of $g$ implies the existence of a $2^{m+1} \cdot 3$-orbit of $\varphi$.

Setting $h=g^{2}=\varphi^{2^{m+1}}$, Lemma 2 and the existence of a 6-orbit of $g$ implies that $h$ has a 3-orbit. Using Lemma 3 (1) and the fact that $\varphi$ has no odd orbit, $\varphi$ has a $2^{m+1} \cdot 3$-orbit.

Following Proposition 2 and Proposition 4, we obtain

Theorem 2. Let an $M-\operatorname{map} \varphi: \mathbb{R} \leadsto \mathbb{R}$ have an $n$-orbit, where $n=2^{m} \cdot q, m \in \mathbb{N}_{0}$, $q$ is odd, and let $n$ be maximal in the Sharkovskii ordering.

1. If $q>3$, then $\varphi$ has a $k$-orbit, for every $k \triangleleft n$, eventually except $k=2^{m+2}$.

2. If $q=3$, then $\varphi$ has a $k$-orbit, for every $k \triangleleft n$, with an eventual exception either for $k=2^{m+1}$ or $k=2^{m+1} \cdot 3,2^{m+2}$.

3. If $q=1$, then $\varphi$ has a $k$-orbit, for every $k \triangleleft n$.

Hence, we can improve Proposition 1 as follows:

Theorem 3. If an $M$-map has an n-orbit, then it has a $k$-orbit for every $k \triangleleft n$ with at most two exceptions.

Corollary 1. If an $M$-map has an $n$-orbit, where $n \neq 2^{m}$, for all $m \in \mathbb{N}$, then infinitely many periodic orbits coexist.

\section{Applichtion to Differential EQUations AND inClusions}

As we have already pointed out, the Poincaré translation operators in (2), associated with continuous differential equations (11), are $M$-maps. Thus, we can recast Theorem 3 and Corollary 1 in the context of subharmonics for equation (1).

Theorem 4. Let all solutions of the continuous differential equation (1), i.e. $F \in$ $C([0,1] \times \mathbb{R}, \mathbb{R})$ and $F(t, x) \equiv F(t+1, x)$, extend to $(-\infty, \infty)$. If (11) has an $n$ periodic solution, then it also admits $k$-periodic solutions, for every $k \triangleleft n$, with at most two exceptions. In particular, if $n \neq 2^{m}$, for all $m \in \mathbb{N}$, then infinitely many (subharmonic) periodic solutions of (11) coexist.

Let us note that Theorem 4 has a meaning in the absence of uniqueness. On the other hand, continuous ordinary differential equations are, according to the result of W. Orlicz $\mathrm{Or}$, generically uniquely solvable. More precisely, the set of continuous functions $F$, for which equation (1) does not have uniqueness of all solutions satisfying the initial conditions $x(0)=x_{0}$, is meager, i.e., a set of the first Baire category. The same result is true for Carathéodory right-hand sides. Therefore, it is perhaps more reasonable to consider differential inclusions instead of differential equations (1). Here, a differential inclusion is given by

$$
x^{\prime} \in F(t, x), \quad F(t, x) \equiv F(t+1, x),
$$

where $F:[0,1] \times \mathbb{R} \leadsto \mathbb{R}$ is an upper-Carathéodory map with nonempty, compact and convex values, i.e.

(i) $F(\cdot, x): \mathbb{R} \leadsto \mathbb{R}$ is measurable (i.e., for any open $U \subset \mathbb{R}$, the set $\{t \in$ $\mathbb{R} \mid F(t, x) \subset U\}$ is measurable, for every $x \in \mathbb{R})$,

(ii) $F(t, \cdot): \mathbb{R} \leadsto \mathbb{R}$ is upper-semicontinuous (i.e., for any open $V \subset \mathbb{R}$, the set $\{x \in \mathbb{R} \mid F(t, x) \subset V\}$ is open, for a.a. $t \in \mathbb{R}$, 
(iii) $|F(t, x)| \leq \alpha+\beta|x|$, for a.a. $t \in \mathbb{R}$ and all $x \in \mathbb{R}$.

The Poincaré translation operators for (3) are defined exactly as for equation (2); they are again $M$-maps (see [AG] and cf. [AFJ]).

Theorem 5. Let $F:[0,1] \times \mathbb{R} \leadsto \mathbb{R}$ be an upper-Carathéodory map (cf. (i)(iii)) with nonempty, compact and convex values. Then the assertion of Theorem 4 remains valid for the inclusion (3).

\section{Concluding Remarks}

Although there are many extensions of Theorem 1-perhaps the most well known is for triangular maps [Kl], until now none of them has been applied to differential equations. Proposition [1 (jointly with the result in [Kl] ) has been generalized to the case where the real line is replaced by a linear continuum and maps are triangular (see $[\mathrm{AP}]$ ). This result can be applied to differential equations and inclusions as in Theorem 5 ]

\section{REFERENCES}

[AFJ] J. Andres, J. Fišer and L. Jüttner: On a multivalued version of the Sharkovskii theorem and its application to differential inclusions. Set-Valued Anal. 10, 1 (2002), 1-14. MR 1888453 (2002m:37057)

[AG] J. Andres and L. Górniewicz: Topological Fixed Point Principles for Boundary Value Problems. Kluwer, Dordrecht, 2003. MR 1998968

[AJ] J. Andres and L. Jüttner: Period three plays a negative role in a multivalued version of Sharkovskii's theorem. Nonlin. Anal. 51 (2002), 1101-1104. MR 1926088 (2003g:37079)

[AJP] J. Andres, L. Jüttner and K. Pastor: On a multivalued version of the Sharkovskii theorem and its application to differential inclusions II. Set-Valued Anal., to appear.

[AP] J. Andres and K. Pastor: On a multivalued version of the Sharkovskii theorem and its application to differential inclusions III. Topol. Meth. Nonlin. Anal. 22 (2003), no. 2, 369386. MR 2036383

[Kl] P. E. Kloeden: On Sharkovsky's cycle coexisting ordering. Bull. Austral. Math. Soc. 20 (1979), 171-177. MR 0557223 (81d:58045)

[Or] W. Orlicz: Zur Theorie der Differentialgleichung $y^{\prime}=f(x, y)$. Bull. Akad. Polon. Sci., Sér. A, 00 (1932), 221-228.

[Pl] V. A. Pliss: Nonlocal Problems in the Theory of Oscillations. Nauka, Moscow, 1964 (in Russian); Academic Press, New York, 1966. MR 0171962 (30:2188)

[Sh] A. N. Sharkovskii: Coexistence of cycles of a continuous map of a line into itself. Ukrainian Math. J. 16 (1964), 61-71 (in Russian). MR 0159905 (28:3121)

Department of Mathematical Analysis, Faculty of Science, Palacký University, Tomkova 40, 77900 Olomouc-Hejčín, Czech Republic

E-mail address: andres@risc.upol.cz

Department of Mathematical Analysis, Faculty of Science, Palacký University, Tomkova 40, 77900 Olomouc-Hejčín, Czech Republic

E-mail address: pastor@inf.upol.cz 